\title{
BMJ Open Witnessing intimate partner violence and child maltreatment in Ugandan children: a cross-sectional survey
}

\author{
Karen M Devries, ${ }^{1}$ Louise Knight, ${ }^{1}$ Jennifer C Child, ${ }^{1}$ Nambusi Kyegombe, ${ }^{1}$ \\ Mazeda Hossain, ${ }^{1}$ Shelley Lees, ${ }^{1}$ Charlotte Watts, ${ }^{1}$ Dipak Naker ${ }^{2}$
}

To cite: Devries KM, Knight L, Child JC, et al. Witnessing intimate partner violence and child maltreatment in Ugandan children: a cross-sectional survey. BMJ Open 2017;7: e013583. doi:10.1136/ bmjopen-2016-013583

- Prepublication history for this paper is available online. To view these files please visit the journal online (http://dx.doi.org/10.1136/ bmjopen-2016-013583)

Received 22 July 2016 Revised 20 October 2016 Accepted 13 December 2016

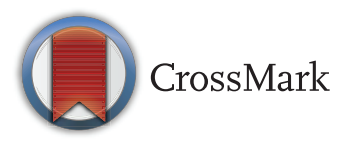

${ }^{1}$ Department of Global Health and Development, London School of Hygiene and Tropical Medicine, London, UK

${ }^{2}$ Raising Voices, Kampala, Uganda

Correspondence to Dr Karen M Devries; karen.devries@Ishtm.ac.uk

\section{ABSTRACT}

Objectives: Existing evidence, mainly from highincome countries, shows children who witness intimate partner violence (IPV) at home are more likely to experience other forms of violence, but very little evidence is available from lower income countries. In this paper we aim to explore whether Ugandan children who witness IPV at home are also more likely to experience other forms of maltreatment, factors associated with witnessing and experiencing violence, and whether any increased risk comes from parents, or others outside the home.

Design: A representative cross-sectional survey of primary schools.

Participants: 3427 non-boarding primary school students, aged about 11-14 years.

Setting: Luwero District, Uganda, 2012.

Measures: Exposure to child maltreatment was measured using the International Society for the Prevention of Child Abuse and Neglect Child Abuse Screening Tool-Child Institutional, and 2 questions measured witnessing IPV.

Results: $26 \%$ of children reported witnessing IPV, but nearly all of these children had also experienced violence themselves. Only $0.6 \%$ of boys and $1.6 \%$ of girls had witnessed partner violence and not experienced violence. Increased risk of violence was from parents and also from other perpetrators besides parents. Both girls and boys who witnessed and experienced violence had between $1.66(95 \% \mathrm{Cl} 0.96$ to 2.87$)$ and $4.50(95 \% \mathrm{Cl}$ 1.78 to 11.33 ) times the odds of reporting mental health difficulties, and $3.23(95 \% \mathrm{Cl} 1.99$ to 5.24$)$ and 8.12 $(95 \% \mathrm{Cl} 5.15$ to 12.80$)$ times the odds of using physical or sexual violence themselves.

Conclusions: In this sample, witnessing IPV almost never occurred in isolation-almost all children who witnessed partner violence also experienced violence themselves. Our results imply that children in Uganda who are exposed to multiple forms of violence may benefit from intervention to mitigate mental health consequences and reduce use of violence. IPV prevention interventions should be considered to reduce child maltreatment. Large numbers of children also experience maltreatment in homes with no partner violence, highlighting the need for interventions to prevent child maltreatment more broadly.

Trial registration number: NCT01678846, results.

\section{Strengths and limitations of this study}

- In high-income settings, there is a wellestablished link between witnessing intimate partner violence and increased risk of exposure to other violence in childhood, but there is limited evidence from low-income countries and on where increased risk of exposure to other violence is coming from.

- This paper provides rare evidence from Uganda, a low-income country, on the relationship between witnessing intimate partner violence and other forms of child maltreatment.

- We are able to explore who the perpetrators of other forms of child maltreatment are, including perpetrators both inside and outside the home.

- We also explore sequelae associated with witnessing and experiencing violence, including mental health and children's risk of using violence against others.

- The study provides valuable first evidence which may help inform intervention targeting, but is limited by its cross-sectional survey design.

\section{INTRODUCTION}

Intimate partner violence (IPV) against women is prevalent globally, with $30 \%$ of women reporting physical and or sexual IPV in their lifetime. ${ }^{1}$ In addition to the known detrimental effects of IPV on women, ${ }^{23}$ witnessing IPV is increasingly being recognised as an important adverse exposure for children. Effects on children include increased risk of depression, anxiety, aggression, conduct disorders, attention deficit and hyperactivity. ${ }^{4}{ }^{5}$ There is evidence that growing up in an abusive family is positively related to future violent intimate relationships. ${ }^{6}$ Estimates from high-income counties indicate that in the range of $8-25 \%$ of adults report exposure to IPV as children. ${ }^{78}$

A growing body of research suggests that children who witness IPV are also at increased risk of being maltreated in other ways. In the USA, nationally representative 
data have shown that $33.9 \%$ of young people who witnessed IPV also experienced another form of maltreatment (neglect, sexual abuse by a known adult, physical abuse and psychological abuse) in the previous year, versus only $8.6 \%$ of young people who did not witness IPV. In the same study other forms of victimisation such as kidnapping, bullying and property crime was also associated with witnessing IPV. ${ }^{9}$ This 'polyvictimisation' ${ }^{9}$ is associated with higher levels of adverse health outcomes versus single exposures. ${ }^{10}$

The extent of the overlap between witnessing IPV and exposure to other forms of maltreatment is not known outside high-income settings, where the epidemiology of violence exposure is likely to be quite different from the USA. ${ }^{11}$ Analyses often do not disaggregate by perpetrator, leaving open the question as to whether parents who have experienced or used IPV also are more likely to use violence against their children, or whether the increased risk of violence is coming from others besides parents. Additionally, studies on the health effects of witnessing IPV often do not account for the fact that witnessing may be correlated with experiencing other forms of violence. $^{9}{ }^{12-14}$

We use baseline data from the Good Schools Study in Uganda to examine (1) the extent of overlap between witnessing IPV between parents, and experience of violence from other perpetrators; (2) whether witnessing is associated with increased risk of violence from parents versus perpetrators besides parents; and (3) factors associated with witnessing violence and experiencing violence. We conducted all analyses separately by sex.

\section{METHODS}

\section{Design}

We analysed baseline survey data from the Good Schools Study, ${ }^{15}$ which is a cluster randomised controlled trial of the Good School Toolkit. The Toolkit was developed by Raising Voices to prevent violence against children in school and improve educational outcomes. The Toolkit is publicly available: http://raisingvoices.org/good-school/, and main trial results are available. ${ }^{16}$

\section{Setting}

The survey took place in Luwero District, Uganda, from June to July 2012. Luwero is near Kampala and has both rural and urban areas.

\section{Sampling}

We obtained a list of all 268 schools registered in Luwero in 2010 from the Ministry of Education and Sports. We excluded 97 small schools (with $<40$ students registered in primary $5(\mathrm{P} 5)$ ) and 20 schools with existing governance interventions. The remaining 151 schools formed our sampling frame. We stratified these 151 schools according to the gender ratio of pupils ( $>60 \%$ girls, $>60 \%$ boys or about even). Forty-two schools were randomly selected, proportional to the size of the stratum. One hundred per cent of the schools agreed to participate. The sampled schools contain $79.7 \%$ of P5, P6 and P7 students in Luwero (equivalent to grades 5, 6 and 7 in the US education system; in Uganda, students in upper primary are about age 11-14 on average). Within each school, we took a simple random sample of up to 130 pupils from P5, P6 and P7 and a complete sample of school staff. If there were $<130$ students in a school, all were invited to participate. Seventy-seven per cent or 3706 sampled students provided data; $19 \%$ were absent from school during the week of the survey or for extended periods. The remaining $4 \%$ were entered on class lists in error, had a parent opt them out, refused or the reason for participation was not recorded. For this analysis, we excluded students who boarded at school, since their patterns of exposure to witnessing and experiencing violence from parents may differ from students who live at home.

\section{Procedure}

For each participating school, headmasters notified staff, students and parents in advance of the survey. Parents could opt their child out of participation; otherwise individual children provided consent to participate. Data were collected in a face-to-face interview. All interviewers received 3 weeks of training on how to ask about violence in a non-judgemental way, preserve confidentiality and procedures if participants became distressed. A comprehensive child protection plan, designed by the study team in conjunction with local services, was in place to provide support to those in need of services. We also had a trained counsellor available to any child who requested counselling.

\section{Instruments}

All items were translated into Luganda and reviewed by a panel of teachers and Raising Voices staff to ensure that they would be appropriate for Ugandan child participants and school staff. Items were then cognitively tested and refined iteratively in a sample of $\sim 40$ children from Kampala primary schools to ensure understanding and that the meanings of original items were adequately captured. We then surveyed a larger sample of 697 children and 40 staff from Kampala schools to test distributions of items and to test study procedures.

Questions related to violence are outlined in table 1 . Witnessing shouting and physical violence between parents/caregivers was measured using two binary response items developed for the study. Experiences of violence were measured by the International Society for the Prevention of Child Abuse and Neglect Child Abuse Screening Tool-Child Institutional (ICAST-CI) ${ }^{17}$ and some items from the WHO Multi Country Study on Women's Health and Domestic Violence against Women. ${ }^{3}$ Reliability and construct validity for the ICAST-CI were initially established in four countries and the instrument has since been translated into 20 languages and used extensively in multicountry research. ${ }^{17}$ 
Table 1 Definitions of violence variables



Lifetime exposure to physical, sexual and emotional violence were constructed as binary variables. Questions related to violence were analysed by perpetrator type (parent/caretaker vs others).

Other measures include demographic variables, use of physical and sexual violence, and mental health. Disability was measured using the following question: 'Do you have any mental or physical disability? For example, do you have trouble seeing, walking, speaking, with fits or anything else?' Responses were grouped into a binary variable, with students who reported any type of difficulty coded as 'disabled'. Symptoms of common childhood mental health difficulties were measured using the Strengths and Difficulties Questionnaire (SDQ). ${ }^{18}$ The SDQ has been used in more than 60 different countries including several in Africa and validated in a variety of settings. ${ }^{18}$ In our sample, reliability for global difficulties scores was Cronbach $\alpha=0.70$. The global SDQ score was constructed as a categorical variable, with children having 'high', 'medium' or 'low' levels of difficulties relative to their peers. To construct this measure responses to 20 items are summed, and children scoring in the highest decile of the overall distribution are deemed to have 'high' difficulties; the next decile to have 'medium' difficulties and the remaining $80 \%$ to have 'low' difficulties. ${ }^{18} 19$

\section{Analysis}

All analyses were conducted using STATA V.12.0 $0^{20}$ (Intercooled Stata [program]. V.12.0. Houston, Texas: Stata Corp, 2012) and were carried out separately for male and female participants. Missing data were excluded from analyses involving those variables (pairwise deletion). In total, $3.8 \%$ of children were missing data on witnessing parents shouting, and $5.2 \%$ were missing data on witnessing physical violence.

Descriptive statistics on participants' background characteristics, witnessing violence and experiencing violence are presented by sex and compared using $\chi^{2}$ tests. When discussing results, we refer to 'students' or 'boys and girls' where findings are similar, and then present separate percentages and highlight where there are differences for boys and girls. We examined overlap between witnessing parental IPV and violence exposure from parents and perpetrators other than parents by fitting logistic regression models adjusted for a priori 
identified potential demographic confounders (tables 2 and 3). Next, we examined factors associated with experience of violence also, and witnessing plus experience of violence, by fitting another set of logistic regression models (tables 4 and 5 ).

All analyses account for the sampling scheme employed in the baseline survey-student responses are weighted to account for unequal probabilities of selection for students. SEs are adjusted for clustering at the school level using Taylor linearisation. ${ }^{20}$

\section{RESULTS}

\section{Demographic characteristics and prevalence of witnessing IPV}

Table 6 describes the characteristics of students and prevalence of witnessing IPV in 3427 non-boarding students surveyed. The majority of students were aged 11-14 years, and more than half of all students had eaten less than three meals in the day before the survey, indicating they were possibly hungry. About $8 \%$ of the students reported some form of disability; more than $65 \%$ of students walked to school with others. Fifty-six per cent of boys but only $15 \%$ of girls indicated that they had ever worked for money, but when examining hours of paid and unpaid work on an average school day, more than half of boys and girls reported working more than 1 hour per day.

About $26 \%$ of boys and girls reported ever witnessing their parents shouting at each other, and $\sim 14 \%$ of boys and girls had ever witnessed physical violence from their mother's male partner towards their mother. Boys and girls differed dramatically on levels of violence experienced from parents or caregivers; however, $9 \%$ of girls but only $5 \%$ of boys reported emotional violence and neglect from parents or caregivers, and $27 \%$ of girls but only $10 \%$ of boys reported physical violence from parents or caregivers. No students reported sexual violence from parents or caregivers.

When examining the overlap in experience of witnessing and experiencing violence (table 6), it becomes clear that witnessing violence almost never occurs in isolation. Less than $1.3 \%$ of children reported witnessing violence without also experiencing violence themselves and $26 \%$ of boys and girls have both witnessed IPV and experienced violence from any perpetrator. About $68 \%$ of boys and $68 \%$ of girls have experienced violence but not witnessed IPV, and only $5.6 \%$ of boys and $4.6 \%$ of girls report never witnessing IPV or experiencing violence.

\section{What forms of violence does witnessing IPV put children at risk for?}

We hypothesised that those who witnessed IPV would be at increased risk of various forms of violence from parents in particular. We found (tables 2 and 3) that girls and boys who witnessed shouting and who witnessed both shouting and physical IPV were at increased risk of emotional and combined emotional and physical violence from parents. Girls were also at increased risk for physical violence, but this association was inconsistent for boys. Boys witnessing shouting and physical IPV had over six times greater odds of experiencing emotional violence or combined emotional and physical violence from parents. Girls who witnessed shouting or shouting and physical IPV had almost four times the odds of combined emotional and physical violence compared with non-witnesses.

However, both boys and girls who witnessed IPV also had increased odds of emotional, physical and sexual violence from perpetrators other than parents. Children who witnessed IPV had between about 1.5 and 3.6 times

Table 2 Associations between violence exposure and witnessing violence between parents, female students

\begin{tabular}{|c|c|c|c|c|c|c|c|c|c|}
\hline \multirow[b]{2}{*}{ Characteristic } & \multicolumn{2}{|c|}{$\begin{array}{l}\text { Female }(n=1423) \\
\text { Witnessed } \\
\text { shouting vs } \\
\text { witnessed no } \\
\text { parental IPV }\end{array}$} & \multirow[b]{2}{*}{ p Value } & \multicolumn{2}{|c|}{$\begin{array}{l}\text { Female }(n=1268) \\
\text { Witnessed } \\
\text { physical IPV vs } \\
\text { witnessed no } \\
\text { parental IPV }\end{array}$} & \multirow[b]{2}{*}{ p Value } & \multicolumn{2}{|c|}{$\begin{array}{l}\text { Female }(n=1364) \\
\text { Witnessed } \\
\text { shouting and } \\
\text { physical IPV vs } \\
\text { witnessed no } \\
\text { parental IPV }\end{array}$} & \multirow[b]{2}{*}{ p Value } \\
\hline & aOR & $95 \% \mathrm{Cl}$ & & aOR & $95 \% \mathrm{Cl}$ & & aOR & $95 \% \mathrm{Cl}$ & \\
\hline \multicolumn{10}{|l|}{ Parental violence } \\
\hline Emotional only & 1.88 & 1.06 to 3.33 & 0.031 & 1.15 & 0.27 to 4.85 & 0.844 & 1.93 & 0.91 to 4.10 & 0.084 \\
\hline Physical only & 1.51 & 1.05 to 2.18 & 0.028 & 1.04 & 0.45 to 2.41 & 0.930 & 1.74 & 1.09 to 2.77 & 0.021 \\
\hline Emotional and physical & 3.90 & 1.95 to 7.81 & $<0.001$ & 3.79 & 1.66 to 8.64 & 0.002 & 3.90 & 1.84 to 8.30 & 0.001 \\
\hline \multicolumn{10}{|l|}{ Non-parental violence } \\
\hline Emotional & 2.65 & 1.84 to 3.81 & $<0.001$ & 1.28 & 0.75 to 2.21 & 0.356 & 2.02 & 1.37 to 2.98 & 0.001 \\
\hline Physical $^{\star}$ & 2.33 & 1.83 to 2.96 & $<0.001$ & 1.68 & 1.00 to 2.81 & 0.049 & 2.54 & 1.82 to 3.54 & $<0.001$ \\
\hline Sexual & 2.36 & 1.49 to 3.76 & 0.001 & 3.19 & 0.67 to 15.14 & 0.139 & 2.98 & 1.81 to 4.89 & $<0.001$ \\
\hline
\end{tabular}


Table 3 Associations between parental violence and witnessing violence between parents, male students

\begin{tabular}{|c|c|c|c|c|c|c|c|c|c|}
\hline \multirow[b]{2}{*}{ Characteristic } & \multicolumn{3}{|c|}{$\begin{array}{l}\text { Male }(n=1334) \\
\text { Witnessed shouting versus } \\
\text { witnessed no parental } \\
\text { violence }\end{array}$} & \multicolumn{3}{|c|}{$\begin{array}{l}\text { Male }(n=1195) \\
\text { Witnessed physical IPV vs } \\
\text { witnessed no parental } \\
\text { violence }\end{array}$} & \multicolumn{3}{|c|}{$\begin{array}{l}\text { Male }(n=1317) \\
\text { Witnessed shouting and } \\
\text { physical IPV vs witnessed } \\
\text { no parental violence }\end{array}$} \\
\hline & aOR & $95 \% \mathrm{Cl}$ & p Value & aOR & $95 \% \mathrm{Cl}$ & p Value & aOR & $95 \% \mathrm{Cl}$ & p Value \\
\hline \multicolumn{10}{|l|}{ Parental violence } \\
\hline Emotional only & 4.28 & 2.20 to 8.31 & $<0.001$ & 0.74 & 0.08 to 6.72 & 0.781 & 6.77 & 3.96 to 11.56 & $<0.001$ \\
\hline Physical only & 1.65 & 0.94 to 2.89 & 0.077 & 1.05 & 0.36 to 3.00 & 0.933 & 0.77 & 0.39 to 1.51 & 0.436 \\
\hline Emotional and physical & 6.53 & 3.29 to 12.96 & $<0.001$ & - & - & & 6.22 & 2.63 to 14.72 & $<0.001$ \\
\hline \multicolumn{10}{|l|}{ Non-parental violence } \\
\hline Emotional & 2.67 & 1.86 to 3.86 & $<0.001$ & 1.48 & 0.83 to 2.62 & 0.175 & 2.87 & 1.72 to 4.78 & $<0.001$ \\
\hline Physical* $^{*}$ & 1.69 & 1.09 to 2.64 & 0.021 & 2.48 & 1.67 to 3.68 & $<0.001$ & 2.34 & 1.52 to 3.56 & $<0.001$ \\
\hline Sexual & 1.84 & 0.94 to 3.61 & 0.074 & 2.24 & 0.57 to 8.83 & 0.243 & 3.51 & 1.52 to 8.10 & 0.004 \\
\hline \multicolumn{10}{|c|}{$\begin{array}{l}\text { aOR is adjusted OR, adjusted for age (years), eaten at least three meals in the past day } \\
\text { children, versus less, share sleeping area with one or more adults versus less, have a d } \\
\text { hours per day versus }<1 \text { hour. } \\
\text { *Any physical violence from non-parents but severe physical violence from school staff. } \\
\text { IPV, intimate partner violence. }\end{array}$} \\
\hline
\end{tabular}

Table 4 Factors associated with experiencing violence and experiencing violence plus witnessing IPV, female students

\begin{tabular}{|c|c|c|c|c|c|c|}
\hline \multirow[b]{2}{*}{ Characteristic } & \multicolumn{3}{|c|}{$\begin{array}{l}\text { Model } 1 \\
\text { Experienced violence from } \\
\text { any perpetrator but did not } \\
\text { witness shouting or physical } \\
\text { parental violence, } n=1190\end{array}$} & \multicolumn{3}{|c|}{$\begin{array}{l}\text { Model } 2 \\
\text { Experienced violence from any } \\
\text { perpetrator and witnessed } \\
\text { shouting or physical parental } \\
\text { violence, } n=2477\end{array}$} \\
\hline & aOR & $95 \% \mathrm{Cl}$ & p Value & aOR & $95 \% \mathrm{Cl}$ & p Value \\
\hline Age (years) & 0.97 & 0.83 to 1.14 & 0.703 & 1.07 & 0.91 to 1.25 & 0.403 \\
\hline Ate at least 3 meals yesterday (vs less) & 1.24 & 0.99 to 1.56 & 0.063 & 0.88 & 0.55 to 1.40 & 0.578 \\
\hline Share sleeping area with 2 or more children (vs less) & 0.68 & 0.53 to 0.89 & 0.005 & 0.93 & 0.65 to 1.33 & 0.674 \\
\hline Share sleeping area with 1 or more adults (vs none) & 1.03 & 0.73 to 1.47 & 0.853 & 1.47 & 0.77 to 2.83 & 0.239 \\
\hline Disability (vs not) & 1.23 & 0.30 to 5.09 & 0.772 & 2.09 & 0.97 to 4.53 & 0.060 \\
\hline Work $<1$ hour per day & 1 & & & 1 & & \\
\hline Work $1-2$ hours per day & 1.52 & 1.08 to 2.15 & 0.017 & 1.72 & 1.04 to 2.86 & 0.036 \\
\hline Work more than 2 hours per day & 3.69 & 1.83 to 7.46 & 0.001 & 4.50 & 1.78 to 11.33 & 0.002 \\
\hline Low SDQ score & 1 & & & 1 & & \\
\hline Medium SDQ score & 2.29 & 1.33 to 3.95 & 0.004 & 3.84 & 1.69 to 8.72 & 0.002 \\
\hline High SDQ score & 1.85 & 0.91 to 3.78 & 0.089 & 4.35 & 1.95 to 9.69 & 0.001 \\
\hline Used physical or sexual violence & 5.14 & 2.99 to 8.84 & $<0.001$ & 8.12 & 5.15 to 12.80 & $<0.001$ \\
\hline
\end{tabular}

the odds of reporting emotional, physical or sexual victimisation by a non-parent or caregiver, versus those who did not witness any violence between caregivers. So it appears that witnessing IPV inside the home is associated with violence both inside and outside the home.

\section{Factors associated with witnessing and experiencing} violence

In tables 4 and 5, we examined the associations between various factors and common patterns of exposure to violence and witnessing IPV. The patterns are: experiencing violence from any perpetrator but not witnessing IPV (model 1), and witnessing IPV plus experiencing violence from any perpetrator (model 2). For both models
1 and 2, associations between demographic factors and violence or violence plus witnessing were similar. However, findings point towards additive effects of witnessing and experiencing violence on mental health and use of violence. Odds of having the higher levels of mental health difficulties and using physical or sexual violence against peers were respectively about two and five times higher in female students who experienced violence versus students who did not experience any violence (model 1). In female students who experienced and witnessed violence, the odds of having higher levels of mental health difficulties and using physical or sexual violence against peers were about four and eight times higher versus students who did not experience or 
Table 5 Factors associated with experiencing violence and experiencing violence plus witnessing, male students

\begin{tabular}{|c|c|c|c|c|c|c|}
\hline \multirow[b]{2}{*}{ Characteristic } & \multicolumn{3}{|c|}{$\begin{array}{l}\text { Model } 1 \\
\text { Experienced violence from } \\
\text { any perpetrator but did not } \\
\text { witness shouting or physical } \\
\text { parental violence } n=1190\end{array}$} & \multicolumn{3}{|c|}{$\begin{array}{l}\text { Model } 2 \\
\text { Experienced violence from any } \\
\text { perpetrator and witnessed } \\
\text { shouting or physical parental } \\
\text { violence } n=598\end{array}$} \\
\hline & aOR & $95 \% \mathrm{Cl}$ & p Value & aOR & $95 \% \mathrm{Cl}$ & p Value \\
\hline Age (years) & 0.88 & 0.79 to 0.97 & 0.015 & 1.01 & 0.85 to 1.21 & 0.887 \\
\hline Ate at least 3 meals yesterday (vs less) & 0.96 & 0.67 to 1.37 & 0.803 & 1.19 & 0.72 to 1.98 & 0.488 \\
\hline Share sleeping area with 2 or more children (vs less) & 0.73 & 0.57 to 0.93 & 0.013 & 0.52 & 0.36 to 0.74 & 0.001 \\
\hline Share sleeping area with 1 or more adults (vs none) & 1.18 & 0.86 to 1.63 & 0.301 & 1.16 & 0.68 to 1.96 & 0.579 \\
\hline Disability (vs not) & 1.49 & 0.91 to 2.44 & 0.114 & 1.12 & 0.51 to 2.46 & 0.775 \\
\hline Work $<1$ hour per days & 1 & & & 1 & & \\
\hline Work $1-2$ hours per day & 1.69 & 1.15 to 2.49 & 0.008 & 1.86 & 1.14 to 3.04 & 0.014 \\
\hline Work more than 2 hours per day & 1.63 & 0.88 to 3.02 & 0.119 & 2.07 & 0.91 to 4.72 & 0.082 \\
\hline Low SDQ score & 1 & & & 1 & & \\
\hline Medium SDQ score & 1.66 & 0.96 to 2.87 & 0.069 & 2.90 & 1.60 to 5.26 & 0.001 \\
\hline High SDQ score & 2.28 & 1.29 to 4.07 & 0.006 & 3.85 & 1.16 to 10.17 & 0.008 \\
\hline Used physical or sexual violence & 3.23 & 1.99 to 5.24 & $<0.001$ & 5.55 & 2.94 to 10.49 & $<0.001$ \\
\hline
\end{tabular}

witness any violence (model 2). For male students, experiencing violence was associated with about two and three times the odds of high mental health difficulties and using physical or sexual violence (model 1), whereas experiencing and witnessing violence was associated with about four and five times the odds of high levels of mental health difficulties and using physical or sexual violence (model 2).

\section{DISCUSSION}

In Luwero district, nearly all students who have witnessed IPV have also experienced emotional, physical or sexual violence themselves. Some of this increased risk of exposure to violence is coming from caregiverswhen there is violence between caregivers, there is also likely to be violence between caregivers and children. However, children who witness IPV are also at increased risk of emotional, physical and sexual violence from other perpetrators outside the home.

The adverse effects of witnessing and experiencing violence are large. Boys and girls who have witnessed and experienced violence have nearly four and five times the odds of having high levels of mental health difficulties, and nearly six and eight times the odds of using violence, versus their boys and girls who have not experienced or witnessed violence. Evidence suggests that witnessing IPV and experiencing violence have additive effects-with children who had witnessed and experienced violence having $\sim 2$ and 3 times the odds of mental health difficulties and using violence, respectively, compared with those experiencing violence alone.

\section{Other studies}

In our sample, the overlap between witnessing IPV and experiencing violence was almost complete-it was extremely rare for children to witness IPV only. This differs somewhat from other representative samples from high-income settings, where witnessing is more common than exposure to maltreatment. ${ }^{9}$ In Uganda, similar to other countries in the region, exposure to violence from various perpetrators including parents, peers and school staff may be more normative and more chronic versus some high-income settings. ${ }^{21-23}$ Further research is needed to fully understand the implications of this, both in terms of the health effects of exposure, and designing appropriate intervention strategies for children in Uganda and similar settings.

In our sample, as in other samples, witnessing and experiencing IPV are strongly associated with poor mental health, and externalising behaviours such as use of violence. ${ }^{24}$ There are various pathways through which witnessing IPV and exposure to violence may contribute to poor mental health. Exposure to emotional, physical and sexual violence can induce a traumatic stress response, which can lead to lasting post-traumatic stress disorder, depression, anxiety and attentional and memory problems. ${ }^{25}$ The direct trauma and stress response of witnessing IPV itself and indirect effect on mothers mental health, ${ }^{26}$ disruption in caregiving due to injuries, economic effects, fathers behaviour and parenting style may all have influence on the child's mental health and well-being. ${ }^{27}$

In high-income settings, evidence suggests that multiple exposures to different forms of violence from different actors has an additive effect on subsequent health risks. ${ }^{10}$ Our evidence is consistent with this pattern, with those who are exposed to witnessing and violence showing very high odds of subsequent mental health difficulties and use of violence. Further research is needed to understand how the differing patterns of violence exposure in settings like Uganda, where some forms of 
Table 6. Characteristics of included students

\begin{tabular}{|c|c|c|c|c|c|c|c|}
\hline \multirow[b]{2}{*}{ Characteristic } & \multirow[b]{2}{*}{$\mathbf{N}^{*}$} & \multicolumn{2}{|l|}{ Male } & \multirow[b]{2}{*}{$\mathbf{N}^{*}$} & \multicolumn{2}{|l|}{ Female } & \multirow[b]{2}{*}{ p Value } \\
\hline & & Per cent & SE & & Per cent & SE & \\
\hline Age & & & & & & & $<0.001$ \\
\hline 10 years or less & 50 & 3.0 & 0.7 & 93 & 5.3 & 1.0 & \\
\hline $11-14$ years & 1275 & 78.7 & 1.6 & 1503 & 84.7 & 1.2 & \\
\hline 15 or more years & 329 & 18.3 & 1.7 & 172 & 10.0 & 1.3 & \\
\hline Number of meals eaten yesterday & & & & & & & 0.032 \\
\hline 1 meal & 225 & 12.7 & 1.2 & 280 & 15.1 & 1.2 & \\
\hline 2 meals & 716 & 41.7 & 2.0 & 644 & 36.7 & 1.6 & \\
\hline $3+$ meals & 715 & 45.6 & 2.7 & 846 & 48.0 & 1.8 & \\
\hline Disability & 122 & 8.0 & 0.7 & 113 & 7.2 & 0.8 & 0.352 \\
\hline Transport to school & & & & & & & 0.019 \\
\hline Other & 96 & 6.1 & 1.6 & 43 & 3.3 & 1.2 & \\
\hline Walking alone & 443 & 28.7 & 3.1 & 447 & 26.9 & 3.1 & \\
\hline Walking with others & 1067 & 65.3 & 3.3 & 1247 & 69.8 & 3.7 & \\
\hline Ever worked for money & 961 & 55.9 & 3.1 & 272 & 14.7 & 1.2 & $<0.001$ \\
\hline Hours worked on average school day & & & & & & & 0.149 \\
\hline$<1$ & 570 & 38.2 & 3.8 & 650 & 38.6 & 2.5 & \\
\hline $1-2$ & 757 & 43.6 & 2.5 & 847 & 47.1 & 2.0 & \\
\hline More than 2 & 327 & 18.2 & 2.3 & 264 & 14.3 & 1.2 & \\
\hline \multicolumn{8}{|l|}{ Violence from parents or caregivers } \\
\hline No violence & 1428 & 87.0 & 1.9 & 1205 & 69.0 & 4.1 & $<0.001$ \\
\hline Sexual violence, lifetime & 0 & 0 & - & 0 & 0 & - & \\
\hline Emotional violence only, lifetime & 48 & 2.7 & 0.6 & 78 & 4.4 & 1.3 & \\
\hline Physical violence only, lifetime & 143 & 8.3 & 1.3 & 395 & 21.9 & 3.3 & \\
\hline Emotional and physical violence, lifetime & 37 & 2.0 & 0.6 & 92 & 4.7 & 0.9 & \\
\hline \multicolumn{8}{|l|}{ Witnessing IPV } \\
\hline No witnessing & 1143 & 73.2 & 1.7 & 1199 & 72.9 & 1.6 & 0.836 \\
\hline Witnessed shouting only, ever & 191 & 12.4 & 1.7 & 225 & 13.3 & 1.0 & \\
\hline Witnessed physical IPV only, ever & 64 & 3.8 & 0.6 & 69 & 4.0 & 0.6 & \\
\hline Witnessed shouting and physical IPV, ever & 174 & 10.7 & 1.0 & 165 & 9.8 & 1.0 & \\
\hline \multicolumn{8}{|l|}{ Violence from non-caregivers } \\
\hline Emotional violence, lifetime & 938 & 58.7 & 2.7 & 993 & 55.8 & 2.9 & 0.485 \\
\hline Physical violence, $\uparrow$ lifetime & 625 & 39.8 & 2.8 & 675 & 39.2 & 2.2 & 0.768 \\
\hline Sexual violence, lifetime & 63 & 3.9 & 0.6 & 239 & 13.3 & 1.3 & $<0.001$ \\
\hline \multicolumn{8}{|l|}{ Witnessing IPV $\ddagger$ and violence from any perpetrator§ } \\
\hline Not witnessed parental IPV or experienced violence & 92 & 5.6 & 0.9 & 72 & 4.6 & 1.6 & 0.446 \\
\hline Witnessed IPV but not experienced any violence & 9 & 0.6 & 0.2 & 24 & 1.3 & 0.5 & \\
\hline Not witnessed IPV but experienced violence & 1051 & 67.6 & 1.8 & 1127 & 68.3 & 1.6 & \\
\hline Witnessed IPV and experience violence & 420 & 26.3 & 1.8 & 435 & 25.8 & 1.7 & \\
\hline
\end{tabular}

${ }^{*}$ Ns are number of participants in each category and are not adjusted for survey design; per cent and SE are weighted and adjusted for clustering.

†ncludes physical violence from any perpetrator but only severe physical violence from school staff.

fWitnessing parental IPV includes shouting or physical IPV.

$\S$ Violence from caregiver or non-caregiver (sexual or emotional violence from any perpetrator and any physical violence from non-parents but severe physical violence from school staff).

IPV, intimate partner violence.

violence, including corporal punishment of children, and IPV against women, may be considered more normative. The influence of context-specific norms on pathways between early violence exposure and later adverse outcomes is largely unknown.

In our sample, there is some suggestion of a sex difference in health effects, where the effects of witnessing and experiencing violence have a stronger relationship with mental health difficulties and use of violence in girls relative to boys. This may be related to the nature of the violence experienced by girls-in our sample, girls are much more likely to report sexual violence, which may have more severe effects relative to other forms of violence exposure. In the USA, witnessing partner violence is associated with a range of different forms of victimisation, but especially increases the risk of being a victim of statutory rape, sexual misconduct and dating violence. ${ }^{9}$ This suggests that witnessing IPV may be associated with having difficult romantic relationships in adolescence. ${ }^{9}$ Further work is needed to understand pathways-it could be that witnessing IPV provides a behavioural model which young people then follow 
when engaged in interpersonal relationships with peers and adults and in their own early romantic relationships.

It is also unclear why children who witness IPV are more likely to experience violence from other perpetrators besides parents and caregivers. Potential mechanisms could include supervision-it could be that children who live in households where parents are in a violent relationship and dysfunction is present have lower levels of supervision and parental support. In the USA, parental supervision can buffer the effects of exposure to violent environments and reduce the risk of violent victimisation for adolescents who have this parental support. ${ }^{28}$ We have also shown that children who witness IPV have a higher risk of mental health difficulties, which includes externalising behaviours, and have a higher risk of using violence themselves. It could be that children who have difficulties at home are more likely to behave disruptively in school and in their communities, which may increase the risk of non-caregiver adults using physical violence to punish their behaviour. Of course, there are also macrolevel factors, including socioeconomic context, poverty and related stress, and cultural and social norms that will shape risk of violence and maltreatment at the level of the family, and the community-individual experiences and behaviours must be seen in the broader socioeconomic and cultural context.

\section{Limitations}

Although we provide some of the first data on witnessing and exposure to violence reported directly by child participants in a low-income setting, our study is not without limitations. Our data are cross-sectional; hence, we are unable to make inferences about causal relationships between witnessing violence, various forms of violence experience, mental health and other factors under study. We used a robust instrument to measure violence exposure; however, we only had two questions to measure witnessing. A more detailed set of questions may have uncovered other types of witnessing experiences which may be important for understanding health outcomes of early exposures to violence. We asked only about witnessing physical IPV from male partners to female caregivers, which may have underestimated prevalence. We also asked about violence from parents and other perpetrators in less detail relative to violence from school staff (as the main objective of our study was to document violence from school staff). This may also have underestimated prevalence. We excluded boarding students, as they may spend substantially less time at home and thus be less exposed to witnessing IPV, hence our results should not be interpreted as generalisable to this group.

In our measure of lifetime physical violence exposure, we included only severe physical violence from school staff, but both more and less severe forms of physical violence from other perpetrators. Physical violence from school staff was overwhelmingly high in our sample (more than $93 \%$ of students reported lifetime experience), hence it would have rendered our exposure measure meaningless if we had used a measure of ever exposure. Further work needs to be done to understand the relationship between different severity levels of violence from different perpetrators with health outcomes. This is a school-based sample so children not attending school, whose experience of witnessing and violence may be different, are not represented.

\section{Implications}

Our study has shown that in our study district, homes with IPV are also highly likely to have child maltreatment. The effects of witnessing and experiencing violence on children results in poor mental health, and greatly increase the odds of use of violence by the child. Our findings suggest that interventions to reduce IPV should be explored for their efficacy in prevention of child maltreatment. One US study of maltreated children showed a decrease in internalising and externalising problems associated with resolution of IPV in the home over time. ${ }^{29}$

Our findings also suggest that many children are experiencing violence in homes where they are not aware of any IPV, suggesting that other child maltreatment prevention strategies are also needed. Programmes which seek to address norms and attitudes about violence against children may change levels of violence, ${ }^{16}$ and programmes which build safe, stable, nurturing and supportive relationships may assist children who have been maltreated or who otherwise have difficulties in achieving better outcomes.

\section{CONCLUSIONS}

Child maltreatment and children's witnessing of IPV between caregivers overlaps substantially, and children who experience both are at greatly increased risk of poor mental health outcomes and externalising behaviour including use of violence against others. Improved understanding of the context-specific epidemiology of multiple and chronic violence exposures in settings like Uganda is needed to help develop and target interventions to reduce child maltreatment and also the adverse consequences associated with it.

Acknowledgements The authors wish to thank the Raising Voices team, their team of interviewers in Luwero District, the participants who so generously gave their time to be part of this work, and their donors.

Contributors KMD conceptualised and performed the analysis, drafted the paper, and obtained funding. LK and JCC managed the Good Schools Study. DN and CW obtained funding. All authors participated in drafting the manuscript.

Funding The Good Schools Study was supported by the UK MRC, DfID and the Wellcome Trust under the Joint Global Health Trials Scheme (MR/ L004321/1 to KMD), and the Hewlett Foundation (to DN). This analysis was supported by the Bernard Van Leer Foundation (to CW).

Competing interests DN developed the Good School Toolkit intervention, which was tested in the Good Schools Study. 
Ethics approval LSHTM (6183) and the Uganda National Council for Science and Technology (SS2520).

Provenance and peer review Not commissioned; externally peer reviewed.

Data sharing statement No additional data are available.

Open Access This is an Open Access article distributed in accordance with the terms of the Creative Commons Attribution (CC BY 4.0) license, which permits others to distribute, remix, adapt and build upon this work, for commercial use, provided the original work is properly cited. See: http:// creativecommons.org/licenses/by/4.0/

\section{REFERENCES}

1. Devries KM, Mak JY, Garcia-Moreno C, et al. Global health. The global prevalence of intimate partner violence against women. Science 2013;340:1527-8.

2. WHO. Global and regional estimates of violence against women: prevalence and health effects of intimate partner violence and non-partner sexual violence. Geneva: WHO, 2013.

3. Garcia-Moreno CJ, Ellsberg $\mathrm{H}$, Heise $\mathrm{M}$, et al. WHO multi-country study on womens health and domestic violence against women. Geneva: WHO, 2005.

4. Martinez-Torteya C, Anne Bogat G, von Eye A, et al. Resilience among children exposed to domestic violence: the role of risk and protective factors. Child Dev 2009;80:562-77.

5. Sternberg K, Baradaran L, Abbott C, et al. Type of violence, age, and gender differences in the effects of family violence on children's behavior problems: a mega-analysis. Dev Rev 2006;26:89-112.

6. Stith SM, Rosen $\mathrm{KH}$, Middleton KA et al. The intergenerational transmission of spouse abuse: a meta-analysis. J Marriage Fam 2000;62:640-54.

7. Wathen CN, MacMillan HL. Children's exposure to intimate partner violence: impacts and interventions. Paediatric Child Health 2013;18:419-21.

8. Gilbert R, Widom CS, Browne K, et al. Burden and consequences of child maltreatment in high-income countries. Lancet 2009;373:68-81.

9. Hamby S, Finkelhor D, Turner $\mathrm{H}$, et al. The overlap of witnessing partner violence with child maltreatment and other victimizations in a nationally representative survey of youth. Child Abuse Negl 2010;34:734-41.

10. Dube SR, Anda RF, Felitti VJ, et al. Childhood abuse, household dysfunction, and the risk of attempted suicide throughout the life span. Findings from the adverse childhood experiences study. JAMA 2001;286:3089-96.

11. Krug E, ed. World report on violence and health. Geneva: WHO, 2002.

12. Graham-Bermann SA, Castor LE, Miller LE, et al. The impact of intimate partner violence and additional traumatic events on trauma symptoms and PTSD in preschool-aged children. J Trauma Stress 2012;25:393-400.

13. Kennedy AC, Bybee D, Sullivan CM, et al. The impact of family and community violence on children's depression trajectories: examining the interactions of violence exposure, family social support, and gender. J Fam Psychol 2010;24:197-207.

14. Finkelhor $\mathrm{D}$, Turner $\mathrm{H}$, Ormrod $\mathrm{R}$, et al. Violence, abuse and crime exposure in a national sample of children and youth. Pediatrics 2009;124:1411-23.
15. Devries KM, Allen E, Child C, et al. The Good Schools Toolkit to prevent violence against children in Ugandan primary schools: study protocol for cluster-randomised controlled trial. Trials 2013;14:232. doi:10.1186/1745-6215-14-23.

16. Devries KM, Knight L, Child JC, et al. The Good School Toolkit for reducing physical violence from school staff to primary school students: a cluster-randomised controlled trial in Uganda. Lancet Glob Health 2015;3:E378-86.

17. International Society for the Prevention of Child Abuse and Neglect. ICAST-C: The ISPCAN Child Abuse Screening Tool- Child Version. Manual and Proposed Guidelines for Pilot Administration: International Society for the Prevention of Child Abuse and Neglect, 2006.

18. Goodman R, Ford T, Simmons $\mathrm{H}$, et al. Using the Strengths and Difficulties Questionnaire (SDQ) to screen for child psychiatric disorders in a community sample. Br J Psychiatry 2000;177:534-9.

19. Department of Health. Strengths and difficulties questionnaire. London, UK: Department of Health, no date.

20. Eltinge JL, Sribney WM. svy1: Some basic concepts for design-based analysis of complex survey data. Stata Tech Bull 1996;31:3-6.

21. Devries KM, Child JC, Allen E, et al. School violence, mental health and educational performance in Uganda. Pediatrics 2014;133: e129-37.

22. UNICEF Tanzania, Centers for Disease Control and Prevention, Muhimbili University of Health and Allied Sciences. Violence against Children in Tanzania: Findings from a National Survey, 2009. Summary Report on the Prevalence of Sexual, Physical and Emotional Violence, Context of Sexual Violence, and Health and Behavioural Consequences of Violence Experienced in Childhood. Dar es Salaam, Tanzania: UNICEF Tanzania, Division of Violence Prevention, National Center for Injury Prevention and Control, Centers for Disease Control and Prevention, Muhimbili University of Health and Allied Sciences, 2011.

23. United Nations Children's Fund Kenya Country Office, Division of Violence Prevention, National Center for Injury Prevention and Control, U.S. Centers for Disease Control and Prevention, Kenya National Bureau of Statistics. Violence Against Children in Kenya. Findings from a 2010 National Survey. Nairobi, Kenya: United Nations Children's Fund Kenya Country Office, Division of Violence Prevention, National Center for Injury Prevention and Control, U.S. Centers for Disease Control and Prevention, Kenya National Bureau of Statistics, 2012

24. MacMillan HL, Wathen $\mathrm{CN}$. Children's exposure to intimate partner violence. Child Adolesc Psychiatr Clin N Am 2014;23:295-308, viii-ix

25. De Bellis MD, Hooper SR, Spratt EG, et al. Neuropsychologica findings in childhood neglect and their relationships to pediatric PTSD. J Int Neuropsychol Soc 2009;15:868-78.

26. Holmes MR. Aggressive behavior of children exposed to intimate partner violence: an examination of maternal mental health, materna warmth and child maltreatment. Child Abuse Negl 2013;37:520-30.

27. Levendosky AA, Lannert B, Yalch M. The effects of intimate partner violence on women and child survivors: an attachment perspective. Psychodyn Psychiatry 2012;40:397-433.

28. Low S, Espelage D. Conduits from community violence exposure to peer aggression and victimization: contributions of parental monitoring, impulsivity, and deviancy. J Couns Psychol 2014;61:221-31.

29. Campbell KA, Thomas AM, Cook LJ, et al. Resolution of intimate partner violence and child behavior problems after investigation for suspected child maltreatment. JAMA Pediatr 2013;167:236-42. 